\title{
The importance of the glycosylation of antimicrobial peptides: natural and synthetic approaches
}

\section{Natalia G. Bednarska, Brendan W. Wren and Sam J. Willcocks}

London School of Hygiene and Tropical Medicine, Keppel Street, London, UK

Glycosylation is one of the most prevalent post-translational modifications of a protein, with a defining impact on its structure and function. Many of the proteins involved in the innate or adaptive immune response, including cytokines, chemokines, and antimicrobial peptides (AMPs), are glycosylated, contributing to their myriad activities. The current availability of synthetic coupling and glycoengineering technology makes it possible to customise the most beneficial glycan modifications for improved AMP stability, microbicidal potency, pathogen specificity, tissue or cell targeting, and immunomodulation.

\section{Introduction}

AMPs are ubiquitous, ancient, and highly effective host defense compounds that are a prominent aspect of the early innate immune response to infection. They vary in sequence and length, but are generally less than 30 amino acids, with a tendency to have a cationic charge that attracts them to bacterial membranes. Their mode of action is also diverse, ranging from direct integration and permeabilisation of the cell wall, binding with nucleic and enzyme targets, to indirect activity, such as immunomodulation of the host. AMPs are often synthesised in an inactive form and then post-translationally cleaved into an active state.

Glycosylation increases the protein and/or peptide diversity and extends their range of functionality. Four distinct types of glycosylation are currently known and being profoundly studied: $\mathrm{N-}, \mathrm{O}-$ $C$-, and $S$-glycosylation. The type of the glycosylation depends on the nature of the sugar-peptide bond and can be further diversified on the base of glycosidic linkage, glycan composition, structure, and length. $N$-type glycosylation, in which glycan is attached to the amino group of asparagine, is well studied. Oligosaccharides attach covalently upon recognition of the Asn-X-Ser/Thr (where X is any amino acid) via a nitrogen atom. Many bacterial glycosyltransferases are already in use for in vitro controlled glycosylation, resulting in the field of protein glycoengineering $[1,2]$.

Corresponding authors: Bednarska, N.G. (natalia.bednarska@Ishtm.ac.uk), Willcocks, S.J. (sam.willcocks@lshtm.ac.uk)
O-linked glycosylation is a dynamically explored field because of its potent role in mammalian pathophysiological processes. Defects in glycosylation in humans have broadly studied links to different diseases and malfunctions [3]. O-linked glycosylation is characterised by the covalent attachment of glycan through an oxygen atom. However, the $\mathrm{O}$-linked consensus, unlike the $\mathrm{N}$ linked one, is not as easily predictable [4]. It is initiated by the attachment of GalNac to Ser/Thr, but can also comprise $O$-linked $\beta$ - $N$-acetylglucosamine; thus, classification of $O$-glycans is based on their initiating monosaccharide [5]. The glycan polymer can vary in heterogeneity, which makes the prediction of glycan building blocks variable in vivo. Additionally, the further branching of the O-glycans involves multiple glycosyltransferases, and our understanding of their function and structure is still in a discovery stage.

Protein $C$-linked glycosylation differs fundamentally from $\mathrm{N}$ and $O$-glycosylation and defines a type of glycosylation in which a carbohydrate is linked to a protein via a carbon atom. In eukaryotes, $C$-glycosylation has been detected in multiple cell lines (e.g., human RNase 2 and IL-12 and rat liver microsomes) [6]. The biological role of $C$-glycosylation is still being elucidated, although the interest of the pharmaceutical industry in the $C$-glycosylation of proteins is related to its unique resistance to metabolic hydrolysis [7]. For $C$-mannosylation, the acceptor sequence comprises $\mathrm{W}$-x-X-W, where the first Trp becomes $C$-mannosylated [8].

$S$-linked glycosylation is the most recent discovery, described for the first time as a post-translational modification of the 
sublancin glycopeptide [9], later also found in other bacteriocins [10]. In $S$-linked glycosylation, the hexose residue is linked to the thiol group of cysteine. An in vitro study of the glycotransferase SunS showed that the enzyme can transfer glucose as well as any hexose to multiple cysteine residues [9]. Therefore, the relaxed substrate specificity of $S$-glycotransferase is similar to that of $N$ glycosylation, and might have wide application in protein and/or peptide engineering.

By 1998, glycosylation had been described in five naturally occurring insect-derived AMPs, all $O$-linked, and proline rich: diptericin, drosocin, formaecin, lebocin, and phyrrorricin [11]. Whereas $O$-linked glycosylation appears to favour proline-rich regions, modifications are sometimes also observed in glycine-rich regions [12]. A curated database of AMPs describes many hundreds of different AMPs from humans, fishes, insects, reptiles, amphibians, molluscs, and crustaceans (http://aps.unmc.edu/AP/main. php). Although most are post-translationally modified in some manner, such as $C$-terminal amidation, cyclisation, or the formation of disulfide bridge(s), only a small fraction, around a dozen or so, are listed as carrying glycosylation motifs (most of these socalled 'gAMPs' are insect derived). Therefore, gAMPs represent a small subclass of AMPs, with broad-spectrum effects [11]. It is becoming clear that the glycosylation of both natural and synthetic AMPs can influence their antimicrobial activity, and their ability to affect host immunity, target specificity, and biological stability [13,14] (Fig. 1).

\section{Glycosylation and antimicrobial activity}

$O$-linked glycosylation of proline-rich AMPs has an important, and often essential, role in their antimicrobial activity. By far the most abundant type of $O$-glycosylation of prokaryotic and eukaryotic peptides is the $\mathrm{N}$-acetylgalactosamine (GalNAc) attachment to serine (Ser) or threonine (Thr) by a glycosidic linkage, as occurs in $O$-mannosylation, $O$-fucosylation, or $O$-glucosylation [15-17].

The importance of glycosylation has been well studied among the insect AMPs, such as diptericin and formaecin and the

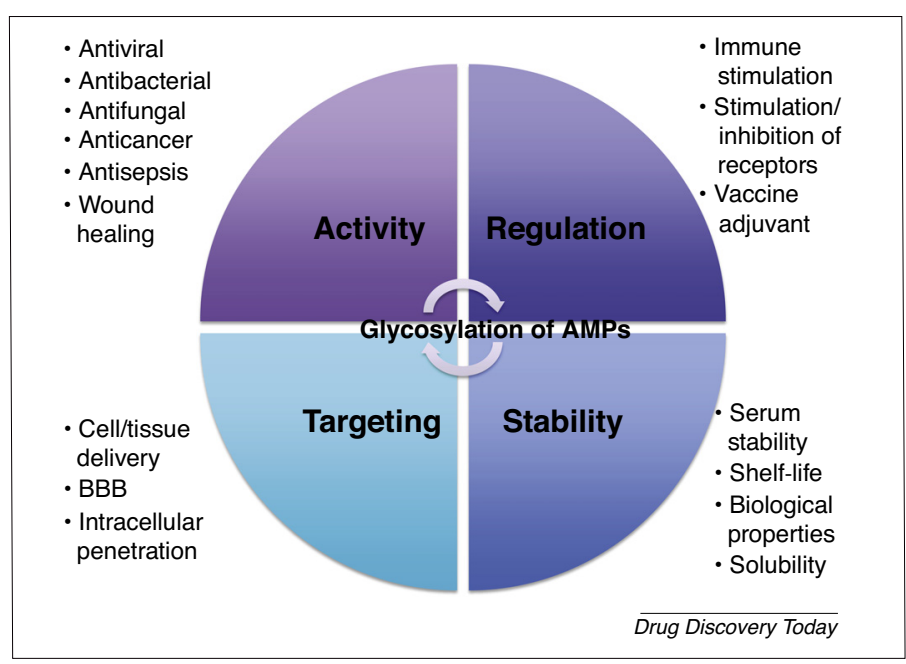

\section{FIGURE 1}

The broad-spectrum effects of glycosylation and its possible utilisation for antimicrobial peptide (AMP) optimisation. Abbreviation: BBB, blood-brain barrier. bacteriocin-family member, enterocin F4-9 [18,19]. In all the above-mentioned peptides, the absence of glycosylation abolishes their antimicrobial activity. Treatment of enterocin with the deglycosylating enzyme, $\mathrm{N}$-acetylglucosaminidase, cleaved the $O$-linked GlcNac linkage from Ser and Thr residues and resulted in a loss of activity [20]. In fish, the glycosylation and glycation of skin gelatin peptides showed enhanced activity against multiple species of bacteria, as well as antioxidant activity [21]. Finally, plants also produce gAMPs to defend against fungal pathogens. One recently identified gAMP, datucin, contains a terminal GlcNac-asparagine, which shows activity against both planktonic and biofilm Candida albicans, and even against multidrug-resistant clinical strains [22].

Evidence of glycosylation affecting the function of human AMP is scarcer, but has been documented [17]. Eosinophil cationic protein (ECP) is exceptionally large compared with typical AMP, but shares many of the same properties with the smaller peptides, such as membrane-lysis activity and affinity towards lipopolysaccharide (LPS) [15]. It is one of many proteins that are presynthesised and stored in granules in readiness to be released upon infection by parasitic invaders. Given that this is a relatively nonspecific process, in the sense that it can be triggered by many different species, diversity among the sequences and the posttranslational modifications of AMP is advantageous [16,17]. Indeed, the level of glycosylation can account for as much as $3 \mathrm{kDa}$ of the total mass of the ECP. Concurrently, damage to the host is a harmful consequence of such broad activity. The extent to which the native form ECP is glycosylated with $N$-linked oligosaccharides is influential. It is currently thought that heavily glycosylation of ECP limits cytotoxicity, and only upon degranulation is the protein deglycosylated to its low-molecular-weight form, which shows greatest cytotoxicity and enhanced lysis of model liposomes [16]. Further evidence that glycosylation of this AMP protects the host from harm comes from the identification of a single nucleotide polymorphism that introduces an additional locus for glycosylation, which reduces cytotoxicity without affecting catalytic activity $[17,23]$.

Glycosylation of AMP does not necessarily result in generation of an efficacious peptide and can sometimes lead to a loss of activity or functionality. As an example, the prokaryotic AMP lysostaphin becomes glycosylated when expressed in mammalian cells, and loses potency against its target, Staphylococcus aureus [24]. Huang et al. identified the precise amino acid glycosylation site on lysostaphin that resulted in the peptide being able to bind, but no longer lyse, $S$. aureus. A single amino acid substitution prevented glycosylation and restored lysostaphin lytic activity [24]. Therefore, it is important to take into consideration the differential expression of peptides among hosts to avoid undesired types of glycosylation [25].

Finally, there are some remarkable examples of AMPs that are not themselves glycosylated but whose function depends upon a larger glycoprotein. Jellein is one such AMP, located within the larger amino acid sequence of major royal jelly protein 1 (MRJP1). Mannosylation of MRJP1-derived peptides facilitates the agglutination of bacteria through interaction with lectin receptors, whereupon jelleins are able to lyse the bacteria in an example of synergy between glycosylated proteins and nonglycosylated peptides [26]. 


\section{Glycosylation and stability}

The glycosylation profile of a peptide not only has a significant role in its antimicrobial properties, but can also improve peptide stability and biological properties both in vitro and in vivo. Table 1 summarises the examples of glycosylated AMPs and the specific effect of their glycosylation, as described in this review.

Several studies have explored the effects of $\mathrm{N}$-glycosylation on peptide rigidity [27], solubility [28], propensity to aggregate [29], conformational changes to the secondary structure of peptides [30], and their ability to influence interactions with neighbouring peptides [31]. This last trait effectively allows glycopeptides to adopt chaperone-like qualities, and has been implicated as having a protective role in preventing fibril formation that contributes to amyloid diseases [29]. More than simply the presence or absence of a glycan, variation in the conformation of the glycosylation affects the stereoisometry of an AMP and can influence its stability. For example, depending on the position of the amino acid that is glycosylated, the linkage of $\alpha$-D-GalNAc-Thr to the AMP, drosocin, alters its rigidity and antibacterial activity [18]. Different types of glycosylation have the ability to influence the rate of fibril formation in prion proteins, where $\mathrm{N}$-glycans reduce the rate of fibrilisation and $O$-glycans affect the rate of folding [28].

The energetics of $\mathrm{N}$-glycan stabilisation is still being studied; however, Levy et al. showed that peptide folding is accelerated because of the intrinsic chemical properties of $\mathrm{N}$-glycans that drive the folding energy landscape by entropic restriction [20]. The thermodynamic stabilisation induced by glycosylation is coupled with kinetic stabilisation [32]. The $\mathrm{N}$-glycan chaperone role is well known to be the stabilising force for disulfide bond formation and proline isomerisation during $\beta$-turns or $\beta$-sheet formation [33]. Peptide stability is an area that has particular prominence in the pharmaceutical synthesis of therapeutic peptides, where it can be affected by factors including $\mathrm{pH}$ and temperature, as well storage and delivery conditions. Peptide glycosylation offers rich possibilities in terms of the degree of glycosylation, and glycan type, structural composition, and size. There are also various options for the site of glycan attachment on the protein, which could bring even greater heterogeneity.

The application of tailored glycosylation of AMP has the potential to modify many biological properties of the peptide in vivo, where $N$-linked glycosylation has been shown to be effective in modifying the serum half-life and bioavailability of peptides and other compounds [34,35]. Enfuvirtide is a novel antiretroviral drug in which glycosylation with sialic acid residues extends its half-life more than tenfold, without affecting its sensitivity towards the target [36]. The pharmacokinetics of glycosylation via the addition of $\mathrm{N}$ - or $\mathrm{O}$-glycans has been found to be similar to the addition of polyethylene glycol moieties (PEGs), which increase the rigidity of AMPs, protecting them from rapid renal clearance [37]. Such modifications aim to increase the size of a given peptide, because molecules with a molecular weight of $<5 \mathrm{kDa}$ that are not associated with plasma proteins tend to be excreted rapidly via the renal system [34]. Glycomodification is preferable to PEG modification because it does not pose the same safety concerns through introduction of a synthetic polymer, and is technically less challenging to achieve [14,38].

Almost all eukaryotic glycoproteins retain a conserved sequence for glycosylation [39]. In vivo, many secreted proteins

TABLE 1

\section{Examples of glycosylated AMPs}

\begin{tabular}{|c|c|c|c|c|}
\hline AMP name & Source & $\begin{array}{l}\text { Natural/synthetic } \\
\text { modification }\end{array}$ & Glycosylation type & Role of glycan \\
\hline Bactenecin & Bovine neutrophils & Natural, synthetic & O-glycosylation & Immunomodulation, regulation, activity \\
\hline Chromacin G & Bovine & Natural & O-linked (NeuAca2-3Galb1-3GalNAca1) & $\begin{array}{l}\text { Antibacterial activity, structural } \\
\text { advantages, proteases resistance }\end{array}$ \\
\hline Datucin & Plant & Natural & O-linked GlcNac & Antifungal Activity \\
\hline Diptericin & Drosophila & Natural & O-linked & Antibacterial activity \\
\hline Drosocin & Insect & Natural, synthetic & $\begin{array}{l}\text { O-linked (a-D-GalNAc-Thr/ } \\
\text { b-DGal(1/3)a-D-GalNAc-Thr) }\end{array}$ & Antibacterial activity \\
\hline Enterocin F4-9 & Bacteria & Natural, synthetic & O-linked & Antibacterial activity \\
\hline $\begin{array}{l}\text { Eosinophil cationic } \\
\text { protein }(E C P)\end{array}$ & Human & Natural & $N$-linked & Activity switch, stability \\
\hline Formaecin & Insect & Natural & O-linked & Antibacterial activity \\
\hline Glycocin F & Bacteria & Natural & $\begin{array}{l}O \text {-linked, } S \text {-linked ( } N \text {-acetylglucosamine } \\
\beta \text {-O-linked to Ser } 18 \text {, and an } \\
N \text {-acetylhexosamine } \\
S \text {-linked to } C \text {-terminal Cys43) }\end{array}$ & Antibacterial activity, stability \\
\hline Lactoferrin h-LF & Human, mammalian & Natural & $\begin{array}{l}\mathrm{N} \text {-linked (highly branched } \mathrm{N} \text {-glycans, } \\
\text { highly sialylated and fucosylated) }\end{array}$ & Stability, regulatory \\
\hline Lebocin & Tobacco hornworm & Natural & O-linked & Antibacterial activity, resistance to proteases \\
\hline Microcin E492 & Bacteria & Natural, synthetic & C-glycosylated moiety & $\begin{array}{l}\text { Evasion of innate immune system, } \\
\text { attack of competing bacteria }\end{array}$ \\
\hline Pyrrhocoricin & Insect & Natural & O-linked & Antibacterial activity \\
\hline Sublancin & Bacteria & Natural & S-linked & Antibacterial activity \\
\hline Tyrocidine A & Bacteria & Natural, synthetic & $N$-linked & Antibacterial activity \\
\hline
\end{tabular}


are co-translationally $\mathrm{N}$-glycosylated with a preformed oligosaccharide at specific amino acid motifs, namely Asn-X-Thr/Ser, before translocation to the endoplasmic reticulum [40]. This natural process has been artificially replicated by the discovery of an amino acid sequence, termed the Enhanced Aromatic Sequon (EAS), which is even more efficiently glycosylated. The EAS can be added to a variety of proteins, and often leads to more rapid protein folding and enhanced stability through better hydrophobic interactions [41].

In bacteria, the $\mathrm{N}$-glycosylation of Asn residues of the AMP, tyrocidine A, produced by the soil bacterium Bacillus brevis, promotes circularisation of this peptide. Studies of synthetic derivatives of tyrocidine A demonstrated the importance of glycosylation, which allows the peptide to fold into its preferential structure without affecting its antimicrobial properties [42]. AMPs, such as bacteriocins, are also more stable in the face of acidic conditions when they are in their circularised conformation, making them attractive for oral administration.

An alternative strategy is to utilise $S$-linked glycosylation, which has been shown to improve the peptide half-life in serum because of its substantial resistance to proteolysis [10]. Therapeutic peptides and proteins face stability issues in vivo partly because of the action of proteolytic enzymes. Glycosylation can help either by masking the peptide from proteinases or by changing the conformation of the peptide to make it more compact or sheltered [43]. In natural systems, $S$-linked glycosylation occurs post-translationally at the cysteine residue, allowing sulfur- $\alpha$-carbon bridges to form, giving conformational strength similar to disulfide bonds. An example is glycocin F, which is an S-linked glycopeptide from the bacteriocin family with a remarkable stability across a wide $\mathrm{pH}$ range (2-10) and resistance to boiling for up to $2 \mathrm{~h}$ [10]. Promisingly, the activity of $S$-glycosyl transferase has been shown to have relaxed substrate specificity, making it an attractive tool for synthetic gAMP design [9]. The considerable advantages offered by the glycosylation of therapeutic peptides highlights the need to develop general approaches to predict the structural consequences of site-specific 'designer' glycosylation. This is rather limited to the trial and error method of testing synthesised glycoproteins. There is a growing market for glycopeptide synthesis, which not only offers help in the design of glycopeptides, but also provides screening and large-scale services (e.g., Bachem and Gly Tech partnership merger).

\section{Glycosylation and target specificity}

Besides their inherent microbicidal properties, the potency of a given AMP is defined by its target specificity. As components of innate immunity, AMPs are a diverse family with a broad-spectrum effect, ranging from different species of bacteria, viruses, and fungi to some cancer cells. Although some AMPs are broad acting, glycosylation offers a means to tailor an AMP to a precise molecular target. Most of the microorganisms rely on glycan-recognition receptors to invade the host cells [44]. Microbes display pathogenassociated molecular patterns (PAMPs) on their surfaces, which are recognised by lectin receptors on the host cells. An understanding of host-pathogen interactions would enable the design of a specific glycosylated AMP that recognises a certain PAMP, specific to a certain microbe, resulting in the highest interspecies selectivity [26].
The ability to modify AMPs to distinguish so carefully between different bacteria has obvious benefits when multiple species are competing for limited resources, and indeed, examples of gAMPs influencing bacterial competition have been observed. For instance, glycocin $\mathrm{F}$ is secreted by Lactobacillus plantarum in the presence of competing bacteria. This peptide has an $\mathrm{O}$-linked $\mathrm{N}$ acteylglucosamine that is essential for its activity with additional $S$-linked $\mathrm{N}$-acetylhexosamine, which increases its bacteriostatic potency [10]. The precise mechanism of action of glycocin $F$ is unknown, but is thought to be result from glycosylation motifs that selectively target lectins displayed by susceptible cells [45]. Plantaricin is another antimicrobial peptide of the bacteriocin family, active against competing lactobacilli and secreted by $L$. plantarum. The lethal mode of action of this peptide results from a selective membrane interaction that causes rapid cell lysis [46]. The exact mechanism of selectivity lies in the preferential affinity of the peptide towards the glycosylated surface proteins of competitors. The removal of the negative charge of the surface glycoprotein reduced the activity of plantaricin, which was dependent on glycosylation patterns displayed on the surface [47]. Other bacteriocins have been shown to interact directly with mannose phosphotransferase system (PTS) transporters found in some bacterial membranes. For example, microcin MccE492 interacts with a surface-expressed mannose transporter on Gramnegative bacteria [48]. Microcin MccE492 is a small $(<10 \mathrm{kDa})$ ribosomal peptide toxin secreted by enterobacteria to enhance host colonisation by preventing the growth of competitive species [49]. Interestingly, the gene cluster encoding microcin MccE492 also enables an unusual post-translational modification, namely an attachment of a monoglycosylated derivative of enterobactin to the MccE492m C-terminus [50]. The result is a C-glycosidic bond that bridges the ribosomal and nonribosomal peptide fragments of Mcc492, shaping the toxin into its active structure. This natural modification is also feasible for a synthetic approach to design new antibiotics specific against Gram-negative species.

Among mammalian AMPs, the bovine peptide, chromacin, is unusual because it carries a net negative, rather than a positive, charge and is hydrophilic, suggesting that it should have poor affinity towards bacterial membranes. However, it is still able to inhibit Gram-positive bacteria [51]. Strub and colleagues revealed that $O$-glycosylation at Ser186 is required for its activity [51]. The glycosylation directs the peptide towards a specific cell surface receptor, overcoming the natural limitations in affinity to bacteria caused by its inherent chemical properties.

Conversely, glycosylation of target sites rather than of the AMPs themselves can represent an important aspect of AMP specificity. In the case of a synthetic AMP, PAF26, glycosylation is a liability for the fungal pathogen, and removal of the fungal glycosylation system enhanced its resistance to PAF26 [52]. Such examples have also been found among non-infectious diseases. The affinity of AMP towards glycoproteins expressed at the host cell surface has been described as a mechanism of selectivity towards cancerous cells, leaving healthy cells unaffected $[47,53]$. Likewise, the cationic AMP, sapecin, containing Lys-Leu-Lys motifs, interacts with negatively charged sialic acid moieties on host eukaryotic membranes [54].

Not all AMPs function by disrupting lipid membranes and causing cell lysis. Proline-rich peptides, such as apidaecin or 
bactenecin, act against intracellular targets. The ability of some AMPs to modify host immunology is at least as valuable as their direct antimicrobial activity. A classic example is the neutrophil AMP, proline-arginine rich peptide (PR-39), which was among the first mammalian nonpore-forming AMPs to be described. It was shown to regulate NADPH oxidase activity, limit excessive tissue damage during inflammation, promote angiogenesis, and aid wound repair $[55,56]$. PR-39 was also implicated in protecting against the metastasis of certain types of cancer by inducing syndecan-1, which is a heparin sulfate proteoglycan involved in the differentiation and prognosis of many types of cancer [57].

Other AMPs have been shown to have diverse systemic effects on the immune system, including cytokine production, and the ability to act as chemokines, to interact with major histocompatibility complexes (MHCs), to stimulate dendritic cells, B cells and T cells [58], and to differentiate macrophages (reviewed in [59]). The specific contribution of glycosylation to these activities is yet to be elucidated, but the propensity for these immune-modifying peptides to be proline rich and commonly associated with glycosylation, suggests an underinvestigated area of research.

\section{Immunomodulation by gAMP}

Different glycosylation patterns are linked with different immune responses, and the $\mathrm{T}$ cell repertoire includes receptors, named $\mathrm{T}_{\text {carb}}$, that specifically recognise glycopeptides complexed with MHC-I and MHC-II [60]. Their specificity towards glycans was shown to be dependent upon the amino acid composition of the $\mathrm{T}$ cell receptor at a specific glycan-recognising region within the $\alpha \beta$ TCR CDR3 region [61].

Interestingly, $\alpha \beta \mathrm{T}_{\text {carb }}$ cells are poor at recognising large, complex glycan structures; therefore, any large branched sugars attached to an antigen would be hidden from recognition by $\alpha \beta \mathrm{T}_{\text {carb }}$ cells [58]. However, larger glycans that are poorly recognised by $\alpha \beta \mathrm{T}_{\text {carb }}$ and MHC-II might be able to directly engage with receptors on B cells and stimulate $\gamma \mathrm{dT}$ cells, which have roles from wound healing to combating tumours and parasitic infections [62]. The direct activation of the B cell receptor by an antigen is most efficient through cross-linking mechanisms that result from recognition of repetitive and ordered structures that are typical of protein aggregates or capsular saccharides [63]. In vaccinology, this effect can be mimicked via the glycosylation of proteins to form a protein-glycan conjugate that promotes B cell receptor clustering, inducing the humoral and memory immunity against the associated pathogen. Early studies using this methodology revealed that the size of the glycan was a crucial aspect in eliciting an effective response, as measured by serum immunoglobulin levels [35]. These myriad properties of glycosylated peptides raise not only concerns, but also opportunities for the rational modification of AMP. Their immunogenicity is an important consideration in the clinical development of new peptides, because immune cells readily generate antibodies against foreign peptide epitopes. This is a problem particularly in repeated dosing, when neutralising antibodies would promote clearance of the peptide or worse, cause adverse inflammatory responses. Indeed, glycosylation of 'self' epitopes to make them appear 'nonself' has been described to contribute to chronic autoimmune diseases, such as type 1 diabetes mellitus and rheumatoid arthritis $[64,65]$.
Peptide immunogenicity can be tailored depending upon the desire to increase or reduce its immunogenicity. For example, $O$ linked or $N$-linked sialic acid residues can have a masking effect against $\mathrm{T}$ cell and B cell receptors [66]. Otherwise, glycosylation can be used to enhance immunogenicity, either in the form of an adjuvant, or as a direct therapy [67]. An excellent example is the design of a glycopeptide that displays high affinity for MHCs and T cell receptors, and is decorated with the same carbohydrate antigen as expressed by tumour cells, the Thomsen-Freidenreich (TF) antigen (beta-Gal-[1 $\rightarrow 3$ ]-alpha-GalNAc-O-serine) [68] to train immunity against a range of cancers; this could represent a future gAMP vaccine.

\section{Rational design of antimicrobial peptides}

In recent years, AMPs have been viewed with both hope and consternation. On the one hand, they offer new tools to combat rising antimicrobial resistance to traditional medication and they are broadly effective against many species at low concentrations. On the other hand, they are limited in terms of their bioavailability and stability in vivo. Glycosylated AMPs could offer the means to finally overcome many of these limitations.

One such a drawback, as well as an advantage, of AMPs is their broad-spectrum activity, which can raise concerns of selectivity between mammalian and bacterial membranes that is largely distinguished because of the ionic differences between these two. Furthermore, AMPs can themselves be immunogenic, or be neutralised by host immune factors. To decrease the reactivity of AMPs within the host, they can be decorated with glycans derived from bacteria, via either chemical conjugation, synthetic approaches, or recombination expression systems. An example of such glycan is polysialic acid, derived from Neisseria meningitidis or Escherichia coli, which renders the peptide less immunogenic. This is because of a structural mimicry with host cell lectins or analogues of sialic acid, which allow the glycosylated peptide to be immune quiescent. The structural mimicry of the $N$. meningitidis glycan to polysialic acid (PSA) suppresses complement deposition and phagocytosis [69]. Overall, because of their enormous diversity and applicability in biopharmaceutical proteins modifications, sialic acids and their analogues represent a separate field of extensive research. Desialylated proteins are rapidly removed from the blood circulation; therefore, conjugating AMP with sialic acid could improve its circulatory half-life. Moreover, sialic acids show potential in the design of blood-brain barrier (BBB)-penetrating peptides, helping to deliver the treatment to the central nervous system. O-linked glycosylation was shown to improve the BBB penetration of an opioid peptide as well as markedly increasing the serum and brain stability of that peptide [70,71]. In the future, BBB-penetrating glycans could be used to help target infections of the nervous system, facilitating elimination of persistent infections and improving therapeutic bioavailability [72].

Moreover, because most AMPs interact with human cell membranes, they could be used as delivery vehicles for bioactive compounds [73]. For example, to deliver an AMP to hepatocytes, one could tag it with a glycan that interacts with the asialoglycoprotein receptor (ASGPR), also known as the hepatic galactose/ $\mathrm{N}$ acetyl-glucosamine receptor [74]. Another example of the useful modification of AMP would be collectin targeting. Collectins contribute to innate immunity by stimulating rapid phagocytosis, 
chemotaxis, and the production of cytokines [75]. There are many different types of organ-specific collectin, such as SP-A and SP-D, which are found in lung and intestine, respectively [76] and which could be excellent targets for the aerosol delivery of AMP in the treatment of lung infections. Peptides with targeted antimicrobial properties are ultimately the main goal to progress most of the currently known AMPs into clinical trials [77].

The utility of using bacterial mimicry of eukaryotic carbohydrates to decorate AMP can also be seen with the $O$-antigen of Helicobacter pylori, which shows targeted interaction with Galectin3 on gastric epithelial cells. The $O$-antigen of $H$. pylori mimics in structure human Lewis blood group antigens, leading to immune avoidance by bacteria so that the host recognises the bacterial antigens as 'self'. $H$. pylori $O$-antigen is decorated with fucose residues and $\mathrm{N}$-acetylneuraminic acid, similar to human sialic acids. Therefore, the rational design of gAMP inspired by this system could aid in both tissue-specific targeting and immune avoidance.

Indeed, knowledge of ligand-receptor interactions can be applied to target gAMP to individual cell types; for example, the interaction between $\mathrm{N}$-acetylgalactosamine with asialoglycoprotein on the surface of hepatocytes [78] or targeting $C$-type lectin receptors to stimulate phagocytosis to target intracellular infection. Delivering gAMP to intracellular targets could also be enhanced by the research undertaken on cell-penetrating peptides (CPPs). This showed that glycans improve the hydrophilic propensities of cationic peptides, without the cost of increased cell cytotoxicity, as sometimes occurs when hydrophobic, balancing amino-acids are introduced [79].

\section{Concluding remarks and future perspectives}

There has been much progress in our understanding of glycobiology in recent years, with applications in vaccinology, cancer biology, and immunology, and potentially revolutionising the field of gAMP design and synthesis. Some antimicrobial peptides are already in clinical use, including ambicin (nisin), polymyxin B, and gramicidin $\mathrm{S}$, with several more in development (reviewed in [80]). The immunomodulatory properties of AMPs have already been applied in what is a conceptually fascinating new tool in the fight against drug-resistant bacteria. Derivatives of the proline-rich bovine AMP, bactenecin, were shown to induce recruitment of immune cells efficient in fighting infection,as demonstrated in vitro [81] and in vivo [82]. Antimicrobial drug development no longer aims to only fight infections, but also to improve systemic health and reduce the general drug toxicity/adverse effects of some antibiotics currently available.

We have emphasised here that glycosylation offers a new aspect to the well-known activity of standard AMPs. Current computer databases containing novel, well-characterised saccharides make it easier to determine which glycan type could be utilised to benefit the overall properties of a therapeutic peptide [83]. Glycan libraries for combinatorial design contain all kinds of glycan, from N/-Oglycans, oligosaccharides, glycosaminoglycans, and mammalian glycans to microbial oligosaccharides [84].

It is also possible to search for glycans with specified backbone types (https://glycosciences.med.ic.ac.uk/glycanLibraryIndex. $\mathrm{html}$ ). A tailored peptide can be visualised in silico in a $3 \mathrm{D}$ form allowing selection of the most beneficial exposure and/or masking of immunogenic epitopes (PDB2multiGif). Moreover, the structure and conformational information can be modelled using the GlycoMaps software, providing additional information before empirical testing [85]. To assess the stability of the peptide and predict its activity, it might be beneficial to determine the influence of glycosylation on hydrophobicity [86] and additional van der Waals interactions [84] (e.g., using Sweet-II [87]). These glycotools can be combined with well-developed tools for the computer-aided design of AMPs and structureactivity modelling software to estimate antimicrobial activity versus quantitative structure-activity relationships (QSAR) $[88,89,90]$.

Such databases facilitate the design of novel gAMPs, from which point there are several synthesis options readily available. The traditional solid-phase synthesis of AMP can produce a peptide that can be chemically conjugated with a glycan $[90,91]$.

Alternatively, the peptides can be expressed by recombinant bacteria, and subsequently glycosylated by plasmid-encoded enzymes. Such glycoengineering offers great potential for the custom glycosylation of target peptides [1]. Mammalian cell line expression systems have also been successfully used, particularly for O-glycosylated peptides [92]. These platforms will inevitably speed up the process of developing new peptide therapeutics, and allow the precise improvement of existing ones. Coupled with high-throughput screening assays of libraries of gAMPs, glycoengineering now offers a relatively fast, cheap, and efficient way to optimise the properties of lead compounds.

The combination of our expanding understanding of the role of natural glycosylation of AMPs in living organisms, the accessibility of large protein and saccharide databases, advanced modelling software, and the huge strides that have been made in glycoengineering, together create the perfect foundation for the design of a new class of therapeutics that hold great potential: glycosylated AMPs.

\section{Acknowledgement}

This work was supported by the U.K. Ministry of Defence.

\section{References}

1 Cuccui, J. et al. (2013) Exploitation of bacterial N-linked glycosylation to develop a novel recombinant glycoconjugate vaccine against Francisella tularensis. Open Biol. 3, 130002

2 Cuccui, J. and Wren, B. (2015) Hijacking bacterial glycosylation for the production of glycoconjugates, from vaccines to humanised glycoproteins. J. Pharm. Pharmacol 67, $338-350$

3 Pinho, S.S. and Reis, C.A. (2015) Glycosylation in cancer: mechanisms and clinical implications. Nat. Rev. Cancer 15, 540-555

4 Hansen, J.E. et al. (1995) Prediction of O-glycosylation of mammalian proteins: specificity patterns of UDP-GalNAc:polypeptide $\mathrm{N}$-acetylgalactosaminyltransferase. Biochem. J. 308, 801-813

5 Peter-Katalinic, J. (2005) Methods in enzymology: O-glycosylation of proteins. Methods Enzymol. 405, 139-171

6 Nishikawa, T. et al. (2008) C-Glycosylation BT. In Glycoscience: Chemistry and Chemical Biology (Fraser-Reid, B.O. et al. eds), pp. 755-811, Springer 
7 Hartmann, S. and Hofsteenge, J. (2000) Properdin, the positive regulator of complement, is highly C-mannosylated. J. Biol. Chem. 275, 28569-28574

8 Doucey, M.-A. et al. (1998) Protein C-mannosylation is enzyme-catalysed and uses dolichyl-phosphate-mannose as a precursor. Mol. Biol. Cell 9, 291-300

9 Oman, T.J. et al. (2011) Sublancin is not a lantibiotic but an $S$-linked glycopeptide. Nat. Chem. Biol. 7, 78-80

10 Stepper, J. et al. (2011) Cysteine S-glycosylation, a new post-translational modification found in glycopeptide bacteriocins. FEBS Lett. 585, 645-650

11 Wang, G. (2012) Post-translational modifications of natural antimicrobial peptides and strategies for peptide engineering. Curr. Biotechnol. 1, 72-79

12 Andreu, D. and Rivas, L. (1998) Animal antimicrobial peptides: an overview. Biopolymers 47, 415-433

13 Opdenakker, G. et al. (1995) Cells regulate the activities of cytokines by glycosylation. FASEB J. 9, 453-457

14 Moradi, S.V. et al. (2016) Glycosylation, an effective synthetic strategy to improve the bioavailability of therapeutic peptides. Chem. Sci. 7, 2492-2500

15 Woschnagg, C. et al. (2009) Eosinophil cationic protein (ECP) is processed during secretion. J. Immunol. 183, 3949-3954

16 Rubin, J. and Venge, P. (2013) Asparagine-linked glycans determine the cytotoxic capacity of eosinophil cationic protein (ECP). Mol. Immunol. 55, 372-380

17 Salazar, V.A. et al. (2014) Protein post-translational modification in host defense: the antimicrobial mechanism of action of human eosinophil cationic protein native forms. FEBS J. 281, 5432-5446

18 Lele, D.S. et al. (2015) Understanding the importance of glycosylated threonine and stereospecific action of Drosocin, a Proline rich antimicrobial peptide. Eur. J. Med. Chem. 92, 637-647

19 Talat, S. et al. (2011) Glycosylated analogs of formaecin I and drosocin exhibit differential pattern of antibacterial activity. Glycoconj. J. 28, 537-555

20 Maky, M.A. et al. (2015) Enterocin F4-9, a novel O-linked glycosylated bacteriocin. Appl. Env. Microbiol. 81, 4819-4826

21 Hong, P.K. et al. (2014) Glycation and transglutaminase mediated glycosylation of fish gelatin peptides with glucosamine enhance bioactivity. Food Chem. 142, 2852 2893

22 Mandal, S.M. (2012) A novel hydroxyproline rich glycopeptide from pericarp of Datura stramonium: proficiently eradicate the biofilm of antifungals resistant Candida albicans. Biopo lymers 98, 332-337

23 Trulson, A. et al. (2007) The functional heterogeneity of eosinophil cationic protein is determined by a gene polymorphism and post-translational modifications. Clin. Exp. Allergy 37, 208-218

24 Huang, C.Y. et al. (2013) Site-specific $N$-glycosylation of caprine lysostaphin restricts its bacteriolytic activity toward Staphylococcus aureus. Anim. Biotechnol. 24, 129-147

25 Harrison, R.L. and Jarvis, D.L. (2006) Protein N-glycosylation in the baculovirusinsect cell expression system and engineering of insect cells to produce 'mammalianized' recombinant glycoproteins. Insect Viruses Biotechnol. Appl. 68, 159-191

26 Brudzynski, K. and Sjaarda, C. (2015) Honey glycoproteins containing antimicrobia peptides, Jelleins of the Major Royal Jelly Protein 1, are responsible for the cell wall lytic and bactericidal activities of honey. PLOS ONE 10, e0120238

27 Wormald, M.R. et al. (1991) The conformational effects of $N$-glycosylation on the tailpiece from serum IgM. Eur. J. Biochem. 198, 131-139

28 Lommerse, J.P. et al. (1995) Conformations and internal mobility of a glycopeptide derived from bromelain using molecular dynamics simulations and NOESY analysis. J. Biomol. NMR 6, 79-94

29 Bosques, C.J. and Imperiali, B. (2003) The interplay of glycosylation and disulfide formation influences fibrillization in a prion protein fragment. Proc. Natl. Acad. Sci. U. S. A. $100,7593-7598$

30 O'Connor, S.E. and Imperiali, B. (1998) A molecular basis for glycosylation-induced conformational switching. Chem. Biol. 5, 427-437

31 Wormald, M.R. and Dwek, R.A. (2016) Glycoproteins: glycan presentation and protein-fold stability. Structure 7, R155-60

32 Shental-Bechor, D. and Levy, Y. (2008) Effect of glycosylation on protein folding: a close look at thermodynamic stabilization. Proc. Natl. Acad. Sci. U. S. A. 105, 8256 8261

33 Shental-Bechor, D. and Levy, Y. (2009) Folding of glycoproteins: toward understanding the biophysics of the glycosylation code. Curr. Opin. Struct Biol. 19, 524-533

34 Byrne, B. et al. (2007) Sialic acids: carbohydrate moieties that influence the biological and physical properties of biopharmaceutical proteins and living cells. Drug Discov. Today 12, 319-326

35 Pozsgay, V. et al. (1999) Protein conjugates of synthetic saccharides elicit higher levels of serum IgG lipopolysaccharide antibodies in mice than do those of the O-specific polysaccharide from Shigella dysenteriae type 1. Proc. Natl. Acad. Sci. U. S. A. 96, 5194-5197
36 Cheng, S. et al. (2015) Glycosylated enfuvirtide: a long-lasting glycopeptide with potent anti-HIV activity. J. Med. Chem. 58, 1372-1379

37 Kozlowski, A. et al. (2001) Development of pegylated interferons for the treatment of chronic hepatitis C. BioDrugs 15, 419-429

38 Pratt, M.R. and Bertozzi, C.R. (2005) Synthetic glycopeptides and glycoproteins as tools for biology. Chem. Soc. Rev. 34, 58-68

39 Lee, H.S. et al. (2015) Effects of $N$-glycosylation on protein conformation and dynamics: Protein Data Bank analysis and molecular dynamics simulation study. Sci. Rep. 5, 8926

40 Aebi, M. (2013) N-linked protein glycosylation in the ER. Biochim. Biophys. Acta Mol. Cell Res. 1833, 2430-2437

41 Price, J.L. et al. (2011) Glycosylation of the enhanced aromatic sequon is similarly stabilizing in three distinct reverse turn contexts. Proc. Natl. Acad. Sci. U. S. A. 108, 14127-14132

$42 \mathrm{Hu}, \mathrm{H}$. et al. (2009) Synthesis and antibacterial activities of $N$-glycosylated derivatives of tyrocidine A, a macrocyclic peptide antibiotic. J. Med. Chem. 52, 2052-2059

43 Solá, R.J. and Griebenow, K. (2010) Glycosylation of therapeutic proteins: an effective strategy to optimize efficacy. BioDrugs 24, 9-21

44 Diebold, S.S. (2009) Activation of dendritic cells by toll-like receptors and C-type lectins. Handb. Ex. Pharmacol. 188, 3-30

45 Brimble, M.A. et al. (2015) Synthesis of the antimicrobial S-linked glycopeptide, glycocin F. Chemistry 21, 3556-3561

46 Wen, L.S. et al. (2016) Purification, characterization and mode of action of plantaricin K25 produced by Lactobacillus plantarum. Food Control 60, 430-439

47 Sand, S.L. et al. (2013) Plantaricin A, a cationic peptide produced by Lactobacillus plantarum, permeabilizes eukaryotic cell membranes by a mechanism dependent on negative surface charge linked to glycosylated membrane proteins. Biochim. Biophys. Acta $1828,249-259$

48 Erni, B. (2006) The mannose transporter complex: an open door for the macromolecular Invasion of bacteria. J. Bacteriol. 188, 7036-7038

49 Donia, M.S. and Fischbach, M.A. (2015) Small molecules from the human microbiota. Science 349, 1254766

50 Nolan, E.M. et al. (2007) Biosynthetic tailoring of microcin E492m: posttranslational modification affords an antibacterial siderophore-peptide conjugate. $J$. Am. Chem. Soc 129, 14336-14347

51 Strub, J.-M. et al. (1996) Antibacterial activity of glycosylated and phosphorylated chromogranin A-derived peptide 173-194 from bovine adrenal medullary chromaffin granules. J. Biol. Chem. 271, 28533-28540

52 Harries, E. et al. (2013) Genes involved in protein glycosylation determine the activity and cell internalization of the antifungal peptide PAF26 in Saccharomyces cerevisiae. Fungal Genet. Biol. 58-59, 105-115

53 Wolfert, M.A. and Boons, G.-J. (2013) Adaptive immune activation: glycosylation does matter. Nat. Chem. Biol. 9, 776-784

54 Weghuber, J. et al. (2011) Cationic amphipathic peptides accumulate sialylated proteins and lipids in the plasma membrane of eukaryotic host cells. Biochim. Biophys. Acta 1808, 2581-2590

55 Gennaro, R. et al. (2002) Pro-rich antimicrobial peptides from animals: structure, biological functions and mechanism of action. Curr. Pharm. Des. 8, 763-778

56 Liu, J. et al. (2016) Cathelicidin PR-39 peptide inhibits hypoxia/reperfusion-induced kidney cell apoptosis by suppression of the endoplasmic reticulum-stress pathway. Acta Biochim. Biophys. Sin. 48, 714-722

57 Ohtake, T. et al. (1999) Proline-rich antimicrobial peptide, PR-39 gene transduction altered invasive activity and actin structure in human hepatocellular carcinoma cells. Br. J. Cancer 81, 393-403

58 Werdelin, O. et al. (2002) Processing of glycans on glycoprotein and glycopeptide antigens in antigen-presenting cells. Proc. Natl. Acad. Sci. U. S. A. 99, 9611-9613

59 Hancock, R.E. et al. (2016) The immunology of host defence peptides: beyond antimicrobial activity. Nat. Rev. Immunol. 16, 321-334

$60 \mathrm{Cobb}, \mathrm{B}$.A. et al. (2004) Polysaccharide processing and presentation by the MHCII pathway. Cell 117, 677-687

61 Speir, J.A. et al. (1999) Crystal structure of an MHC class I presented glycopeptide that generates carbohydrate-specific CTL. Immunity 10, 51-61

$62 \mathrm{Wu}$, Y.L. et al. (2014) gammadelta T cells and their potential for immunotherapy. Int. J. Biol. Sci. 10, 119-135

63 Treanor, B. (2012) B-cell receptor: from resting state to activate. Immunology 136 , $21-27$

64 Maverakis, E. et al. (2015) Glycans in the immune system and the altered glycan theory of autoimmunity: a critical review. J. Autoimmun. 57, 1-13

65 Marth, J.D. and Grewal, P.K. (2008) Mammalian glycosylation in immunity. Nat. Rev. Immunol. 8, 874-887

66 Bork, K. et al. (2009) Increasing the sialylation of therapeutic glycoproteins: the potential of the sialic acid biosynthetic pathway. J. Pharm. Sci. 98, 3499-3508 
67 Zimmermann, S. and Lepenies, B. (2015) Glycans as vaccine antigens and adjuvants: immunological considerations. Methods Mol. Biol. 1331, 11-26

68 Xu, Y. et al. (2004) Designer glycopeptides for cytotoxic T cell-based elimination of carcinomas. J. Exp. Med. 199, 707-716

69 Fujita, T. et al. (1999) Inhibitory effect of free sialic acid on complement activation and its significance in hypocomplementemic glomerulonephritis. J. Clin. Lab. Anal. 13, 173-179

70 Egleton, R.D. et al. (2001) Improved blood-brain barrier penetration and enhanced analgesia of an opioid peptide by glycosylation. J. Pharmacol. Exp. Ther. 299, $967-$ 972

71 Witt, K.A. et al. (2001) Peptide drug modifications to enhance bioavailability and blood-brain barrier permeability. Peptides 22, 2329-2343

72 Papademetriou, I.T. and Porter, T. (2015) Promising approaches to circumvent the blood-brain barrier: progress, pitfalls and clinical prospects in brain cancer. Ther. Deliv. 6, 989-1016

73 Reinhardt, A. and Neundorf, I. (2016) Design and application of antimicrobial peptide conjugates. Int. J. Mol. Sci. 17, 701

74 Shi, B. et al. (2013) Expression of asialoglycoprotein receptor 1 in human hepatocellular carcinoma. J. Histochem. Cytochem. 61, 901-909

75 Lehr, C.-M. and Gabor, F. (2004) Lectins and glycoconjugates in drug delivery and targeting. Adv. Drug Deliv. Rev. 56, 419-420

76 McCormack, F.X. and Whitsett, J.A. (2002) The pulmonary collectins, SP-A and SP$\mathrm{D}$, orchestrate innate immunity in the lung. J. Clin. Invest. 109, 707-712

77 Brogden, N.K. and Brogden, K.A. (2011) Will new generations of modified antimicrobial peptides improve their potential as pharmaceuticals? Int. J. Antimicrob Agents 38, 217-225

78 Roggenbuck, D. et al. (2012) Asialoglycoprotein receptor (ASGPR): a peculiar target of liver-specific autoimmunity. Auto-Immunity Highlights 3, 119-125

79 Kramer, J.R. et al. (2015) Reinventing cell penetrating peptides using glycosylated methionine sulfonium ion sequences. ACS Cent. Sci. 1, 83-88
80 Fox, J.L. (2013) Antimicrobial peptides stage a comeback. Nat. Biotechnol. 31, 379-382

81 Niyonsaba, F. et al. (2013) The innate defense regulator peptides IDR-HH2, IDR-1002, and IDR-1018 modulate human neutrophil functions. J. Leukoc. Biol. 94, 159-170

82 Scott, M.G. et al. (2007) An anti-infective peptide that selectively modulates the innate immune response. Nat. Biotechnol. 25, 465-472

83 Powell, A.K. et al. (2010) Generating heparan sulfate saccharide libraries for glycomics applications. Nat. Protoc. 5, 821-833

84 Mazola, Y. et al. (2011) Integrating bioinformatics tools to handle glycosylation. PLoS Comput. Biol. 7, e1002285

85 Kumar, S. et al. (2012) GlycoCD: a repository for carbohydrate-related CD antigens. Bioinformatics 28, 2553-2555

$86 \mathrm{Lu}$, D. et al. (2012) How hydrophobicity and the glycosylation site of glycans affect protein folding and stability: a molecular dynamics simulation. J. Phys. Chem. B 116 390-400

87 Bohne, A. et al. (1998) W3-SWEET: carbohydrate modeling by internet. Mol. Model. Апnu. 4, 33-43

88 Cherkasov, A. et al. (2014) QSAR Modeling: where have you been? Where are you going to?. J. Med. Chem. 57, 4977

89 Frank, M. et al. (2002) Rapid generation of a representative ensemble of $\mathrm{N}$-glycan conformations. Silico Biol. 2, 427-439

90 Zhou, Q. et al. (2014) Site-specific antibody-drug conjugation through glycoengineering. Bioconjug. Chem. 25, 510-520

91 Rachel, N.M. and Pelletier, J.N. (2016) One-pot peptide and protein conjugation: a combination of enzymatic transamidation and click chemistry. Chem. Commun. 52 2541-2544

92 Hossler, P. et al. (2009) Optimal and consistent protein glycosylation in mammalian cell culture. Glycobiology 19, 936-949 